\section{Non-inhibitory antibodies inducing increased emicizumab clearance in a severe hemophilia A inhibitor patient}

Hemophilia A is a bleeding disorder that results from coagulation factor VIII (FVIII) deficiency, which can be treated via substitution therapy using FVIII concentrates. However, the generation of neutralizing antibodies renders treatment ineffective in up to $30 \%$ of the severe patients. ${ }^{1,2}$ Emicizumab is a humanized bispecific antibody that binds simultaneously to activated factor IX (FIXa) and factor X (FX), thereby mimicking the cofactor function of activated factor VIII (FVIIIa), even in the presence of FVIII inhibitors. ${ }^{3,4}$ Once-weekly subcutaneous administration of emicizumab markedly decreased the bleeding rate in patients who had hemophilia A with or without FVIII inhibitors. ${ }^{5-8}$ However, anti-drug antibodies with neutralizing potential can develop in a small number of patients and have been associated with decreased emicizumab plasma concentrations and loss of efficacy. ${ }^{9,10}$ Although the development of neutralizing antiemicizumab antibodies is rare and routinely biological monitoring is not recommended in patients treated with emicizumab, it is still important to detect the presence of such antibodies in case of bleeding events. In this study, we describe the development of non-inhibitory anti-emicizumab antibodies that selectively provoke increased emicizumab clearance in a severe hemophilia A patient with inhibitors.

A 2-year-old boy with severe hemophilia A developed an anti-FVIII inhibitor $(1 \mathrm{BU} / \mathrm{mL})$ at 19 exposure days. The patient failed to respond to immune tolerance induction and venous access became extremely complicated.
Treatment with emicizumab was therefore initiated with four loading doses $(3 \mathrm{mg} / \mathrm{kg} /$ week) followed by weekly treatment $(1.5 \mathrm{mg} / \mathrm{kg} /$ week $)$. Clinical outcomes were excellent with no bleeding episodes or bruising. Blood samples were taken as part of routine care, with excess being stored for research (The Hôpital Necker's hemophilia bio-library/Necker Biobank, registration number: DC-2009-955; procedure is in accordance with the Helsinki declaration and participants gave written informed consent). Analysis revealed emicizumab concentrations in the expected range $(66 \mu \mathrm{g} / \mathrm{mL} 52$ days after emicizumab initiation), ${ }^{5}$ and a dramatic decrease in activated partial thromboplastin time (APTT) ratio (0.74, normal range $<1.2$ ) was measured (Figure 1).

A spontaneous hemarthrosis of the ankle occurred 6 months after emicizumab initiation, which was confirmed via clinical examination and ultrasound evaluation. Simultaneously, the APTT-ratio rose to 2.67, and circulating emicizumab concentrations were below $1 \mu \mathrm{g} / \mathrm{mL}$ (Figure 1). Hence, the development of anti-emicizumab antibodies was suspected, and the presence of emicizumab-specific immunoglobulin G (IgG) in the patient's serum was analyzed in immunosorbent assays, using normal serum and IgG-depleted patient serum as controls. Binding of $\mathrm{IgG}$ to immobilized emicizumab $(5 \mu \mathrm{g} / \mathrm{mL})$ was determined using isotype-specific peroxidase-labeled monoclonal antibodies. Whereas no specific IgG2 or IgG3 anti-emicizumab antibodies were detected, the patient's serum was indeed enriched in anti-emicizumab antibodies of the IgG1 subtype (Figure 2A and $\mathrm{B}$ ). We could not test for IgG4 antibodies, since emicizumab is of this subtype. ${ }^{3}$

Treatment of the hemarthrosis included rFVIIa and oral

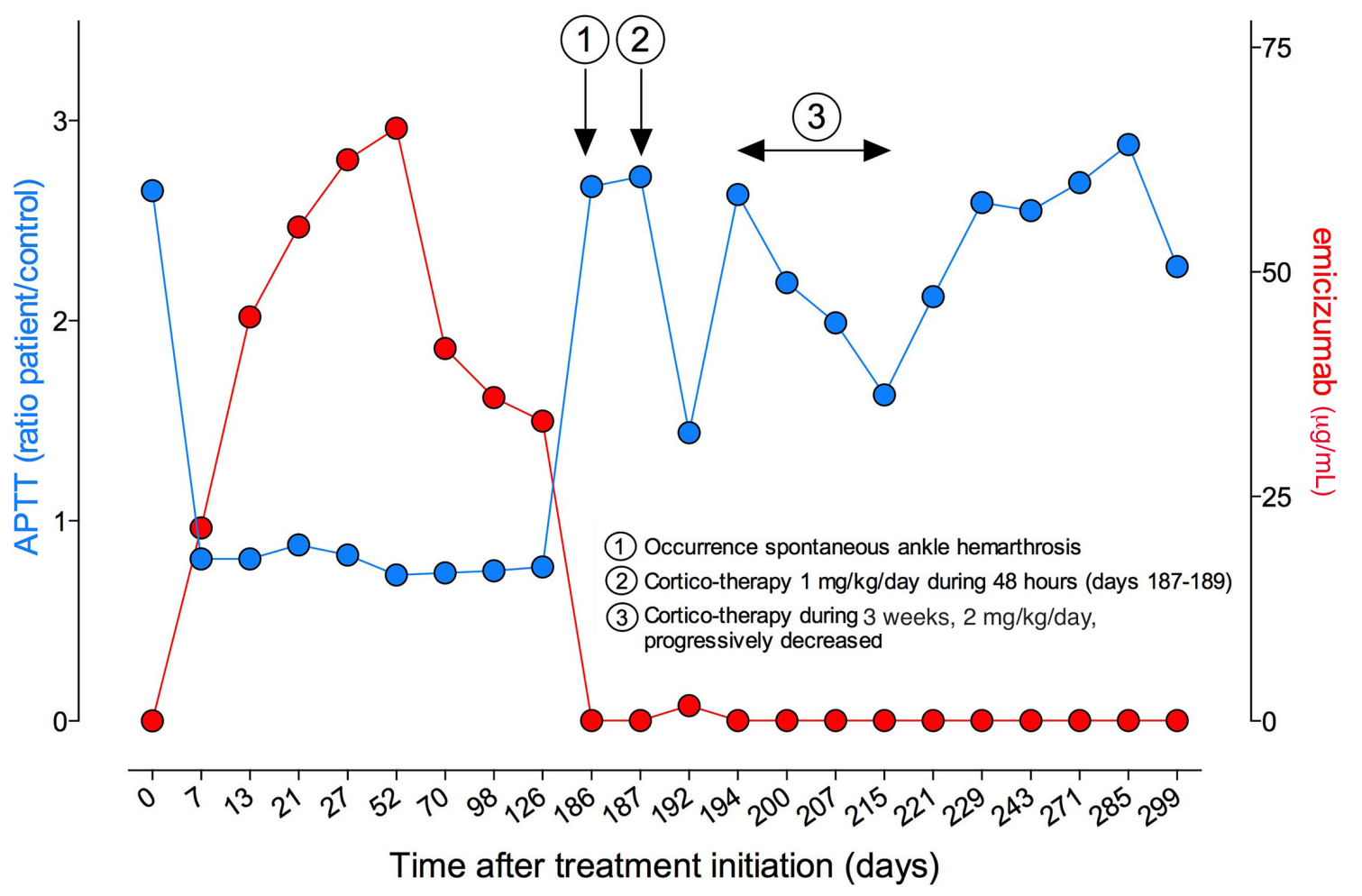

Figure 1. Evolution of APTT and emicizumab plasma concentration over time. At indicated time points, blood samples were taken from the patient. Plasma was then analyzed for APTT (left Y-axis, blue circles) and emicizumab concentration (right Y-axis; red circles). Arrow 1 indicates bleeding event; arrows 2-3 indicate periods of cortico-therapy (2: $1 \mathrm{mg} / \mathrm{kg} /$ day for 48 hours during day 187-189; $3: 2 \mathrm{mg} / \mathrm{kg} /$ day during 3 weeks during days $194-214$, with progressive decrease in dosing). ATPP: activated partial thromboplastin time. 
B

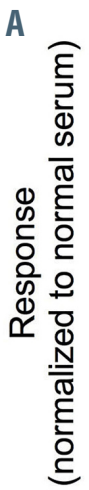

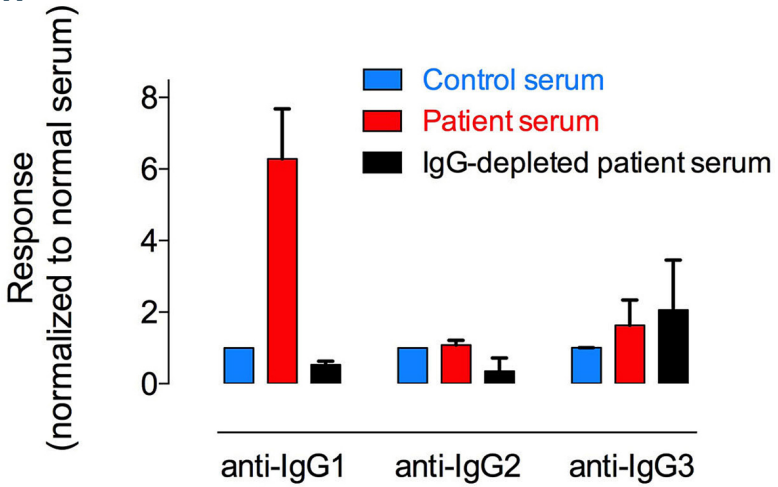

C

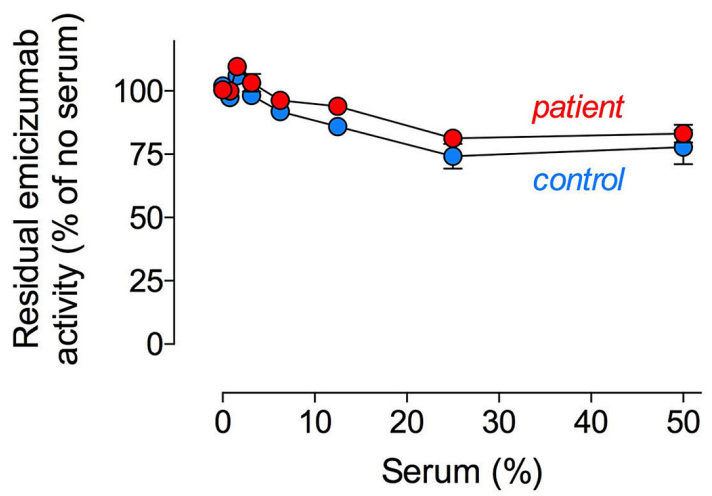

E

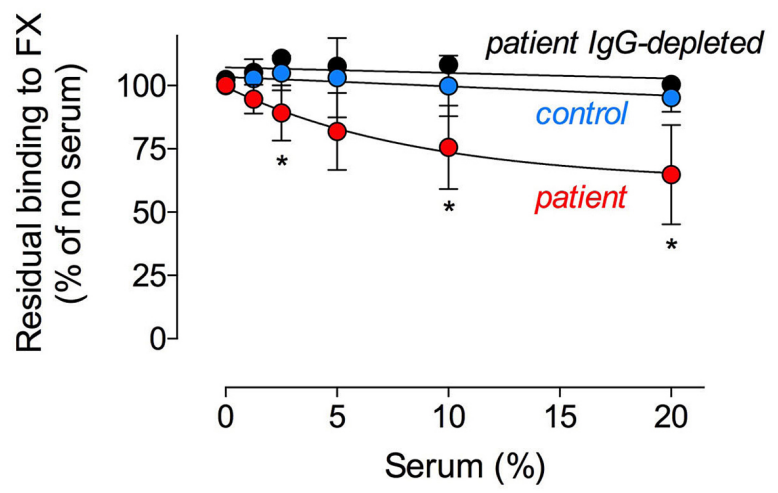

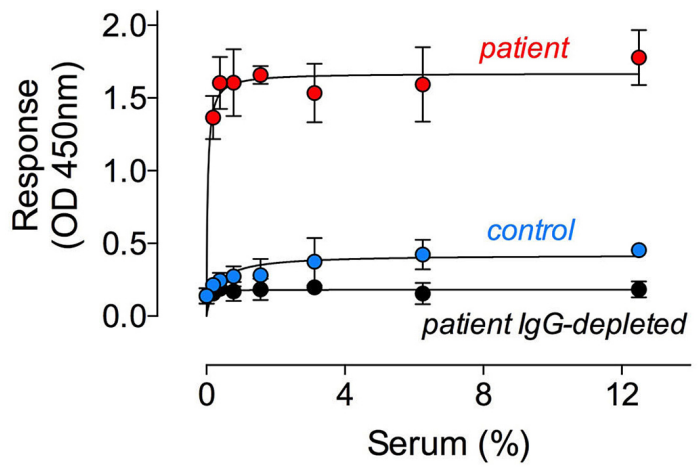

D

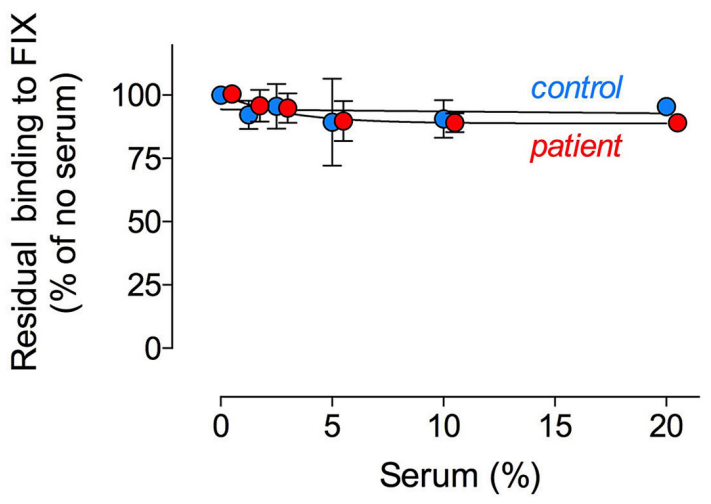

$\mathbf{F}$

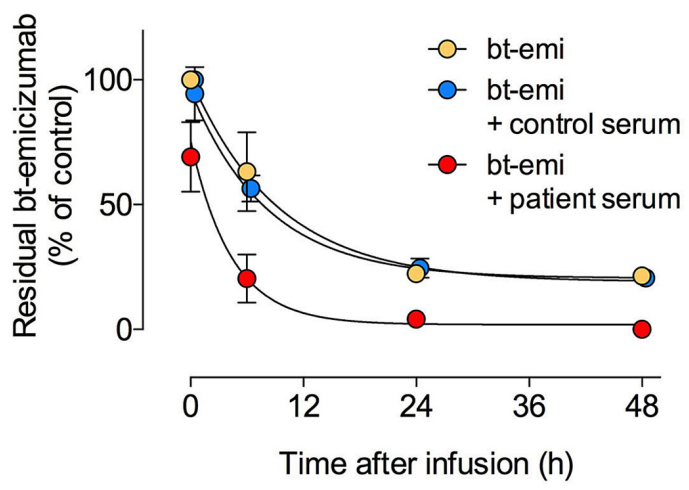

Figure 2. Characterization of anti-emicizumab antibodies. (A and B) Emicizumab was immobilized (5 $\mu \mathrm{g} / \mathrm{mL}$ ) and incubated with control serum (blue), patient serum (red) or immunoglobulin G (IgG)-depleted patient serum (black). Bound anti-emicizumab antibodies were probed using peroxidase-labeled IgG-subtype
specific antibodies, and detected via 3,3',5,5'-tetramethyl benzidine (TMB) hydrolysis. For panel A, samples were diluted 256 -fold, and response was normalized specific antibodies, and detected via 3,3,5,5'-tetramethyl benzidine (TMB) hydrolysis. For panel A, samples were diluted 256-fold, and response was normalized
to that of normal plasma, which was arbitrarily set at 1. For panel B, the dose-response for binding of IgG1 antibodies to emicizumab is shown. (C) Emicizumab to that of normal plasma, which was arbitrarily set at 1 . For panel B, the dose-response for binding of lgG1 antibodies to emicizumab is shown. (C) Emicizumab
$(25 \mathrm{\mu g} / \mathrm{mL})$ was incubated in the absence or presence of various dilutions of control serum (blue circles) or patient serum (red circles). Presented is the percentage residual emicizumab activity relative to the absence of serum as measured in a chromogenic factor VIII (FVIII)-activity assay using human FIXa and factor $X(F X)$. (D) Binding of bt-emicizumab $(50 \mu \mathrm{g} / \mathrm{mL})$ to immobilized factor IX (FIX) $(5 \mu \mathrm{g} / \mathrm{mL})$ was performed in the absence or presence of various dilutions of control serum (blue circles) or patient serum (red circles). Bound bt-emicizumab was probed with peroxidase-labeled streptavidin and detected via TMB hydrolysis. Shown is the percentage residual FIX binding relative to the absence of serum. (E) Binding of bt-emicizumab $(10 \mu \mathrm{g} / \mathrm{mL})$ to immobilized FX ( $5 \mu \mathrm{g} / \mathrm{mL}$ ) was performed in the absence or presence of various dilutions of control serum (blue circles) or patient serum (red circles). Bound bt-emicizumab was probed with peroxidase-abed ment was plo serum and patient serum. (F) Immuno-deficient mice received bt-emicizumab $(0.25 \mathrm{mg} / \mathrm{kg}$ ) alone (orange circles) or in the presence of control serum (100 $\mu \mathrm{L}$ blue circles) or patient serum ( $100 \mu \mathrm{L}$; red circles) via intravenous injection in the retro-orbital plexus. At indicated time points, samples were taken and plasma was analyzed for the presence of residual bt-emicizumab. Presented is the percentage of residual bt-emicizumab relative to bt-emicizumab alone at 3 minutes after injection, which was arbitrarily set at $100 \%$. Lines were generated by fitting the data to an equation describing a single-exponential decay. Data represent the mean \pm standard deviation of three experiments. 
corticoids $(1 \mathrm{mg} / \mathrm{kg} /$ day for 48 hours $[\mathrm{h}$ ] to reduce periarticular inflammation). A minor increase in emicizumab concentrations $(1.7 \mu \mathrm{g} / \mathrm{mL})$ and reduction in APTT-ratio (1.44) was observed (Figure 1), suggesting a potential cortico-sensitivity of the anti-emicizumab antibody-producing plasmocytes. Although no bleeds were observed during a 3-week period, emicizumab levels remained undetectable following a short corticosteroid therapy ( $2 \mathrm{mg} / \mathrm{kg} / \mathrm{day}$, conform to the management of children's immunologic thrombocytopenic purpura). Corticosteroid-therapy was therefore halted. Since antiemicizumab antibodies have been reported to be transient in some patients, ${ }^{11}$ emicizumab therapy $(1.5 \mathrm{mg} / \mathrm{kg} /$ week) was continued for 3 months. As no improvement was observed, emicizumab therapy was terminated.

In order to further characterize the anti-emicizumab antibodies, additional tests were performed. We next analyzed eventual inhibition of emicizumab activity using the chromogenic activity assay (Biophen FVIII:C activity assay [ref 221402]; Hyphen BioMed, Andresy, France). Surprisingly, no reduction in emicizumab activity was observed, irrespective of whether normal or patient serum was tested (Figure 2C). Similar data were obtained using a one-stage clotting assay, suggesting that the antiemicizumab antibodies are essentially non-inhibitory.

This was further assessed by analyzing binding of biotinylated (bt)-emicizumab to immobilized purified recombinant FIX (Pfizer, Paris, France) or plasma-derived FX (Cryopep; Montpellier, France). Biotinylation was performed using the NHS-PEO4-biotin kit (ref: UPR2027B; Griener Bio-one; Frickenhausen, Germany) without loss of cofactor activity. No inhibition of emicizumab binding to FIX was observed with normal or patient serum (Figure 2D). However, a modest inhibition of emicizum$\mathrm{ab}$ binding to FX was observed using patient serum (Figure 2E). Maximal inhibition was $35 \pm 20 \% \quad(n=4$, $P=0.0124$ compared to normal serum) using 5 -fold diluted serum. The absence of a dominant inhibitory effect on the interactions between emicizumab and FIX/FX is compatible with the non-inhibitory nature of the patient's anti-emicizumab antibodies in the activity assays. Indeed, a modest decrease in emicizumab-FX complex formation would still allow for sufficient ternary complex to be formed (555 pM vs. $851 \mathrm{pM}$ in the absence of inhibitor, when calculated according to Kitazawa et al.). ${ }^{12}$ We previously showed that a threshold of about 300 pM ternary complex is needed to produce sufficient hemostatic activity in vivo. ${ }^{13}$

As low emicizumab levels in the patient can be explained by an increased clearance, we determined survival of bt-emicizumab in the absence or presence of serum in immuno-deficient mice. Bt-emicizumab was added to $0.9 \% \mathrm{NaCl}, 100 \mu \mathrm{L}$ control serum or $100 \mu \mathrm{L}$ patient serum (both $5 \mu \mathrm{g}$ per $100 \mu \mathrm{L}$ ), and incubated for 30 minutes $(\mathrm{min})$ at ambient temperature. Mixtures were then injected via the retro-orbital plexus (at a dose of 0.25 $\mathrm{mg} / \mathrm{kg}$ bt-emicizumab) to male NOD.CB17Prkdcscid/NCrHsd-mice (age 9 weeks; ENVIGO, Gannat, France). Housing and experiments of NOD.CB17Prkdcscid/NCrHsd-mice were performed in accordance with French regulations and the experimental guidelines of the European Community. Experimentation was approved by the local ethical committee of the Universite Paris-Sud (Comité d'Éthique en Experimentation Animale no. 26, protocol APAFIS\#44002016021716431023v5). Blood samples were taken at 15 min, $6 \mathrm{~h}, 24 \mathrm{~h}$ and $48 \mathrm{~h}$ after infusion. Plasma was used to determine residual bt-emicizumab levels in an enzyme-linked immonosorbent assay (ELISA) in which streptavidin-coated microtiter wells were incubated with plasma samples, and bound bt-emicizumab was probed using peroxidase-labeled anti-human IgG4-Fc antibodies. No difference in the disappearance from the circulation was found between bt-emicizumab alone or the bt-emicizumab/normal serum combination $\left(t_{1 / 2}=8.5 \mathrm{~h}\right.$ and $8.3 \mathrm{~h}$, respectively; Figure $2 \mathrm{~F}$ ). In contrast, bt-emicizumab was eliminated significantly faster in the presence of patient serum $\left(\mathrm{t}_{1 / 2}=2.2 \mathrm{~h} ; P=0.0013\right.$; Figure $\left.2 \mathrm{~F}\right)$, with no bt-emicizumab detectable at $48 \mathrm{~h}$ after infusion (compared to $21 \%$ for both other conditions). It, thus, seems that the main effect of the anti-emicizumab antibodies is to accelerate clearance of emicizumab.

Contrary to previously reported inhibitors of emicizumab, we here describe the presence of antibodies that leave emicizumab activity unaffected, but that provoke the rapid elimination of emicizumab in mice, providing an explanation for the low emicizumab levels in the patient. Although the occurrence of anti-emicizumab antibodies is rare, their presence may severely diminish its clinical efficacy, resulting in the re-appearance of spontaneous bleeds. Clinical monitoring will usually be sufficient for patients receiving emicizumab therapy. However, in case of bleeding in the absence of any compliance concerns, biological monitoring (APTT and emicizumab concentration) is therefore helpful to detect possible anti-drug antibodies.

Annie Harroche, ${ }^{1, *}$ Thibaud Sefiane, ${ }^{2, *}$

Maximilien Desvages, ${ }^{2,3}$ Delphine Borgel, ${ }^{2,3}$ Dominique Lasne, ${ }^{2,3}$ Caterina Casari, ${ }^{2}$ Ivan Peyron, ${ }^{2}$ Laurent Frenzel, ${ }^{1}$ Stéphanie Chhun, ${ }^{4}$ Peter J. Lenting ${ }^{2}$ and Cécile Bally'

Centre de Traitement de l'Hémophilie, AP-HP, Hôpital Necker Enfants Malades, Paris; 'Laboratory for Hemostasis, Inflammation \& Thrombosis, Unité Mixed de Recherche 1176, Institut National de la Santé et de la Recherche Médicale, Université Paris-Saclay, Le Kremlin-Bicêtre; ${ }^{3}$ Laboratoire d'Hématologie, AP-HP, Hôpital Necker Enfants Malades, Paris and 'Laboratoire d'Immunologie Biologique, AP-HP, Hôpital Necker Enfants Malades, Paris, France

*AH and TS contributed equally as co-first authors.

Correspondence:PETER J.LENTING - peter.lenting@inserm.fr

doi:10.3324/haematol.2021.278579

Received: February 15, 2021.

Accepted: May 3, 2021.

Pre-published: May 20, 2021.

Disclosures: AH received fees for consultancy and board expert membership from Roche, Takeda, LFB, CSL-Behring, Sobi and NovoNordisk; DL received consulting fees from Roche (with fees going to Association de Recherche en Hématologie à Necker-Enfants Malades; ARHNEM); PJL received speaker fees from Biotest, Chugai, CSL-Behring, LFB-Biomedicament, NovoNordisk, Roche, Sanofi, Takeda and research support from Sobi, Catalyst Biosciences. All other authors declare no conflicts of interest.

Contributions: $A H, C B$ were in charge of the patients and provided the clinical samples; $M D, D B$ and $D L$ performed the coagulation tests; TS, CC, IP and PJL designed and performed the in vitro and in vivo experiments. All authors contributed to the interpretation of the data. $A H, C B$ and PJL wrote the first draft of the manuscript, and all authors contributed to the editing of the final manuscript.

Acknowledgments: we thank Dr. Alyanakian MA who leads the biological Necker Biobank.

Data sharing statement: data are available upon reasonable request to the corresponding author. 


\section{References}

1. Cormier M, Batty P, Tarrant J, Lillicrap D. Advances in knowledge of inhibitor formation in severe haemophilia $\mathrm{A}$. Br J Haematol. 2020;189(1):39-53.

2. Scott DW, Pratt KP. Factor VIII: perspectives on immunogenicity and tolerogenic strategies. Front Immunol. 2019;10:3078.

3. Kitazawa T, Igawa T, Sampei Z, et al. A bispecific antibody to factors IXa and X restores factor VIII hemostatic activity in a hemophilia A model. Nat Med. 2012;18(10):1570-1574.

4. Sampei Z, Igawa T, Soeda T, et al. Identification and multidimensional optimization of an asymmetric bispecific IgG antibody mimicking the function of factor VIII cofactor activity. PLoS One. 2013; 8(2):e57479.

5. Oldenburg J, Mahlangu JN, Kim B, et al. Emicizumab prophylaxis in hemophilia A with inhibitors. N Engl J Med. 2017;377(9):809-818.

6. Mahlangu J, Oldenburg J, Paz-Priel I, et al. Emicizumab prophylaxis in patients who have hemophilia A without inhibitors. $\mathrm{N}$ Engl Med. 2018;379(9):811-822.

7. Young G, Liesner R, Chang T, et al. A multicenter, open-label phase 3 study of emicizumab prophylaxis in children with hemophilia A with inhibitors. Blood. 2019;134(24):2127-2138

8. Pipe SW, Shima M, Lehle M, et al. Efficacy, safety, and pharmacokinetics of emicizumab prophylaxis given every 4 weeks in people with haemophilia A (HAVEN 4): a multicentre, open-label, non-randomised phase 3 study. Lancet Haematol. 2019;6(6):e295-e305.

9. Harkins Druzgal C, Kizilocak H, Brown J, Sennett M, Young G. Neutralizing antidrug antibody to emicizumab in a patient with severe hemophilia A with inhibitors: new case with detailed laboratory evaluation. J Thromb Haemost. 2020;18(9):2205-2208.

10. Valsecchi C, Gobbi M, Beeg M, et al. Characterization of the neutralizing anti-emicizumab antibody in a patient with hemophilia $\mathrm{A}$ and inhibitor. J Thromb Haemost. 2021;19(3):711-718.

11.Paz-Priel I, Chang T, Asikanius E, et al. Immunogenicity of emicizumab in people with hemophilia A (PwHA): results from the HAVEN 1-4 studies. Blood. 2018;132(Suppl 1):S633.

12. Kitazawa T, Esaki K, Tachibana T, et al. Factor VIIIa-mimetic cofactor activity of a bispecific antibody to factors IX/IXa and X/Xa, emicizumab, depends on its ability to bridge the antigens. Thromb Haemost. 2017;117(7):1348-1357.

13. Ferriere S, Peyron I, Christophe OD, et al. A hemophilia A mouse model for the in vivo assessment of emicizumab function. Blood. 2020;136(6):740-748. 